\title{
Indoor Location System for Security Guards in Subway Stations
}

\author{
Juan Francisco De Paz ${ }^{1}$, Gabriel Villarrubia ${ }^{1}$, Javier Bajo ${ }^{2}$, \\ Gabriel Sirvent ${ }^{3}$, and Tiancheng $\mathrm{Li}^{4}$ \\ ${ }^{1}$ Department of Computer Science and Automation, University of Salamanca, \\ Plaza de la Merced, s/n, 37008, Salamanca, Spain \\ \{gvg, fcofds\}@usal.es \\ ${ }^{2}$ Department of Artificial Intelligence. Faculty of Computer Science . \\ Technical University of Madrid, Madrid, Spain \\ jbajo@fi.upm.es \\ ${ }^{3}$ Intelligence Artificielle, University of Toulouse, \\ 15 Rue des Lois, 31000 Toulouse France \\ sirventgabriel@gmail.com \\ ${ }^{4}$ School of Mechatronics, Northwestern Polytechnical University, \\ Xiniversity, China \\ t.c.li@mail.nwpu.edu.cn
}

\begin{abstract}
Indoor locating systems (RTLS), have notably advanced during recent years, becoming one of the main challenges for several research teams. The main objective of indoor locating systems is to obtain functional systems able to locate different elements in those environment where GPS (Global Positioning System) is limited. The growing use of mobile devices in the information society provides a powerful mechanism to obtain geographical data and has led to new algorithms aimed at facilitating object positioning with easonable power consumption. In this paper we propose an innovative indoor location architecture that makes use of the data provided by mobile devices to locate objects. The architecture is applied to a case study in a real environment focused on obtaining the location of security staff in the subway network in a city in the north of Spain.
\end{abstract}

Keywords: indoor locating system, Wi-Fi, MQTT.

\section{Introduction}

Indoor locating systems have significantly evolved during recent years [10] [13] [15] [3]. The main challenge of current research in indoor locating systems is to provide a system that can calculate the position of different resources in indoor systems with a reduced cost [1][2]. Estimating the location of a given resource constitutes the basis of the design of advanced services such as resource identification, security, 
recommendation systems, human behaviour analyzers, etc. [28][30]. The majority of these services are deployed in indoor environments such as hospitals, subway stations, shopping malls and so on, characterized by a weak or inexistent GPS signal. Thus, taking into account the importance of location-based services[11][14], it is necessary to design new and effective indoor locating systems.

Nowadays, the use of Wi-Fi technology allows the development of real time indoor locating systems with a low-cost infrastructure[16][17] compared to alternative technologies such as (Radio Frequency iDentification) [5] or ZigBee[6]. The technological infrastructure required to provide indoor location use Wi-Fi communication, which is based on the deployment of Access Points in the environment to be controlled. These access points can be used as readers. One of the advantages of this kind of solution is that Wi-Fi systems are very common and it is usual to find Wi-Fi[20][22] access points installed in a broad variety of public places, hospitals or environments dedicated to leisure activity. The existing technology[18] can be reused to implement indoor location solutions without additional costs. Furthermore, the use of wireless technologies makes it possible to locate people by means of their smartphones or another electronic low-cost devices.

In this paper we propose an indoor location architecture based on Wi-Fi technology that provides the location of the subway security guards and determines the station where the security guard is located at any given moment. The systems calculates the position of the security guard based on his or her personal smartphone[23][12][27], which presents several limitations including connectivity problems and battery consumption. The smartphone is not only used as a tag to be located, but also as a way to comminicate with other security guards. The architecture defines a new algorithm to estimate the real positon of the users using GSM (Global System for Mobile) signals and resource-constrained devices. The algorithm uses reduced data frames and the infrastructure uses a low-latency setting, which facilitates effective communications with an external server.

The rest of the paper is organized as follows: Section 2 revises the state of the art of the situation that has motivated this research, Section 3 presents the architecture and its application to a case study. Finally Section 4 present the results obtained.

\section{Background}

Nowadays it is possible to find differnt algorithms and commercial systems aimed at facilitating the indoor location of a user. Table 1 presents a comparative study between the main existing systems, identifying their strengths and weaknesses.

Existing systems [19] [21] present business models with several restrictions, based on pay-per-use systems and hardware renting systems. 
Table 1. Comparison Between Commercial Systems

\begin{tabular}{|c|c|c|c|c|c|c|}
\hline DEVICE & THECNOLOGY & TYPE & SIZE (CM) & $\begin{array}{l}\text { ACCURACY } \\
\text { (M) }\end{array}$ & ADVANTAGES & $\begin{array}{c}\text { DISADVANTAG } \\
\text { ES }\end{array}$ \\
\hline $\begin{array}{l}\text { HELICOMM } \\
\text { (EZ-TRACER) }\end{array}$ & ZigBee & Beacons & $6.8 \times 4 \times 1.7$ & 3 & $\begin{array}{c}\text { Development } \\
\text { Kit }\end{array}$ & Very Expensive \\
\hline EKAHAU & WiFi & Tag & $4.5 \times 5.5 \times 1.9$ & 5 & Through wall & $\begin{array}{c}\text { Calibration } \\
\text { Process }\end{array}$ \\
\hline AEROSCOUT & WiFi-RFID-UWB & Beacons & $7.4 \times 5 \times 1$ & 15 & $\begin{array}{l}\text { Movement } \\
\text { sensor }\end{array}$ & $\begin{array}{c}\text { Calibration } \\
\text { Process }\end{array}$ \\
\hline UBISENSE & $\begin{array}{c}\text { UWB 7Ghz-TDOA- } \\
\text { AOA }\end{array}$ & Beacons & $8.3 \times 4.2 \times 1.1$ & 0.3 & - & $\begin{array}{l}\text { Without } \\
\text { Warranty }\end{array}$ \\
\hline $\begin{array}{c}\text { SAPHIRE } \\
\text { MULTISPECTR } \\
\text { AL } \\
\end{array}$ & TDOA 5.94Ghz & Tag & $\begin{array}{c}2.8 \times 2.8 \times 2.5 \\
4\end{array}$ & 1 & Only USA & - \\
\hline AWARE POINT & ZigBee & Tag & $1.6 \times 2.7 \times 0.5$ & 10 & ZigBee Alliance & Very Expensive \\
\hline TIMEDOMAIN & UWB & Tag & $1.9 \times 2.3 \times 0.9$ & 15 & $\begin{array}{l}\text { Health Enviro- } \\
\text { ment }\end{array}$ & $\begin{array}{c}\text { Poor Informa- } \\
\text { tion }\end{array}$ \\
\hline PLACELAB & WiFi & Beacons & $1.9 \times 2.8 \times 1.9$ & 13 & Open Source & Microsoft \\
\hline
\end{tabular}

The common feature of all the systems evaluated [25] [29] (and main disadvantage) is the introduction of proprietary hardware, which does not make is possible to use a conventional smartphone to identify location. Furthermore, many existing systems require [24] [26] the use of a tag component. This item type is invasive for users and lacks functionality if a similar service can be offered by using a mobile terminal.

\section{Location Architecture for Subway Environments}

This section presents an arquitecture especially designed to locate human resources in indoor environments, more specifically in subway stations. The special characteristics of this environment require the design of an innovative solution. The main objective of the proposed architecture is to locate security staff in subway stations. Different alternatives have been evaluated to design the architecture. The proposed system uses GSM signals and smartphones, due to the execessive cost that the use of wi-fi tags can cause. The system infrastructure, which is shown in Figure 1, is composed of two different sub-systems: central server and mobile devices. From the software point of view the architecture proposes two elements: the location engine and the location system, both of which are explained in the following sub-sections.

\subsection{Communication System}

This system interconnects the mobile devices with a central server, which is in charge of calculating positions from the information acquired by the mobile devices. 


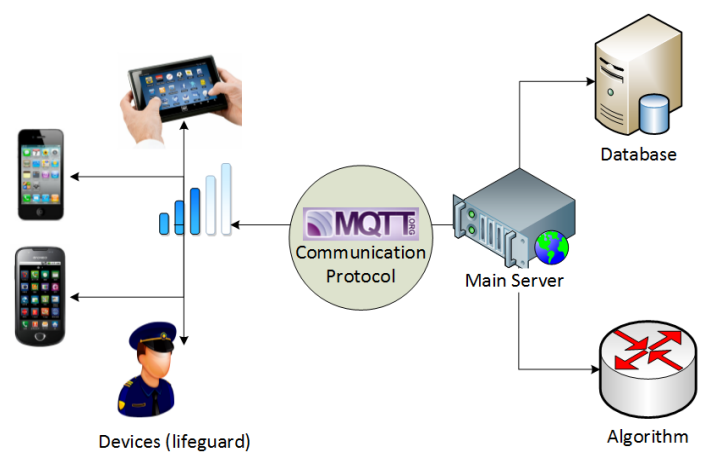

Fig. 1. Proposed architecture

Mobile Devices: An Android application is installed in each of the mobile devices executed as a service. The application periodically scans the wireless networks detected in the surroundings and sends the information collected to the central server. The data frame format contains the information of the detected networks, the time of the scan, the mobile device identifier and the percentage of battery of the device.

The Wi-Fi networks detected by the mobile devices correspond to the access points installed in the subway stations. The Wi-Fi infrastructure for each of the subway stations consists of two Access points, model UBN PICOSTATION M2H, on either side of the platform. The stations have a length of $100 \mathrm{~m}$ while the Access points can cover $150 \mathrm{~m}$.

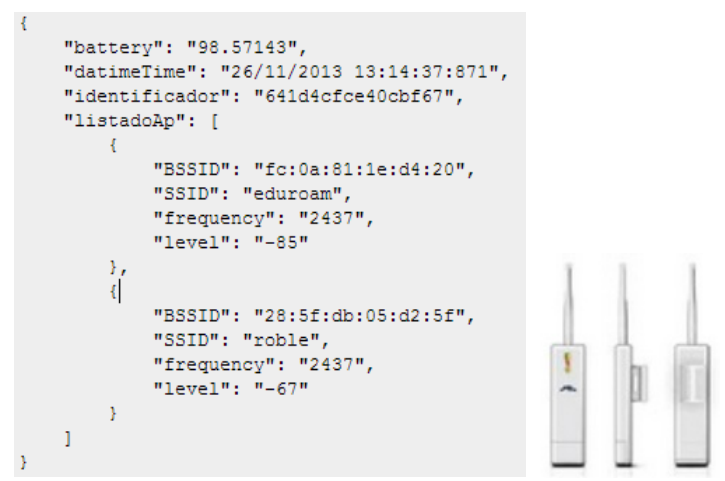

Fig. 2. Mobile Datagram Example. Example of Beacon

Central Server: The mobile devices capture the data which are sent to a central server using an innovative communication system called MQTT (MQ Telemetry Transport). The use of a communication protocol as Machine-to-Machine (M2M) allows data transmission in high latency networks with certain constraints [31]. It is a lightweight protocol that allows a publication-subscription mechanism, which is very 
useful for a low consumption bandwidth. In the architecture presented in this paper, the subway stations are not equipped with a high speed bandwidth and the communication between the mobile devices and the central server is implemented by means of GPRS (General Packet Radio Service).

One of the most innovative aspects of the proposed architecture [32] is the integration within a MQTT protocol for Information exchange. This provides high efficiency compared to the existing commercial systems that use SOA (Service Oriented Architecture). The comparison shown in Table 2 shows underscores the advantages of using a MQTT protocol instead of a traditional client/server architecture in terms of battery consumption. Table 2 presents a comparison between MQTT and HTTPS messages for a total of 1024 communications [7].

Table 2. Comparative Battery saving

\begin{tabular}{|c|c|c|c|c|}
\cline { 2 - 5 } \multicolumn{1}{c|}{} & \multicolumn{2}{c|}{3 M } & \multicolumn{2}{c|}{ WiFi } \\
\cline { 2 - 5 } \multicolumn{1}{c|}{} & HTTPS & MQTT & HTTPS & MQTT \\
\hline $\begin{array}{c}\% \text { Battery / } \\
\text { Hour }\end{array}$ & $18.43 \%$ & $16.13 \%$ & $3.45 \%$ & $4.23 \%$ \\
\hline $\begin{array}{c}\text { Messages } \\
\text { Hour }\end{array}$ & 1708 & 160278 & 3628 & 263314 \\
\hline $\begin{array}{c}\text { \% Battery / } \\
\text { Message }\end{array}$ & 0.01709 & 0.00010 & 0.00095 & 0.00002 \\
\hline $\begin{array}{c}\text { Messages } \\
\text { Received }\end{array}$ & $240 / 1024$ & $1024 / 1024$ & $524 / 1024$ & $1024 / 1024$ \\
\hline
\end{tabular}

As can be seen in Table 2, the use of MQTT can be justified in terms of battery savings alone.

\subsection{Location System}

The location algorithm presented in this paper uses a signpost technique to calculate the station where a security guard is located [4]. Signpost is characterized by its simplicity and a low computational cost [8]. The location of each of the security guards is estimated detecting the more powerful signal as shown in Figure 4.

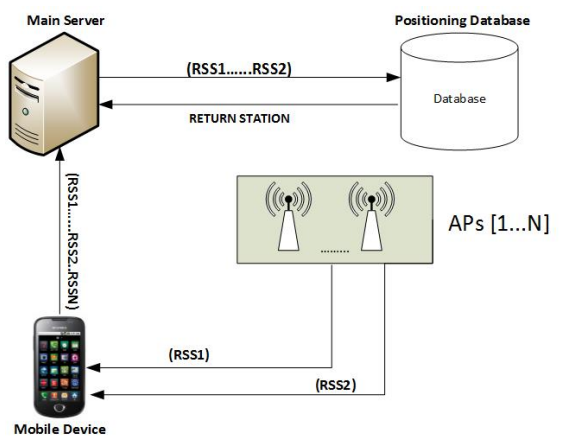

Fig. 3. Wifi Architecture 
As seen in Figure 5, the 12 subway stations in the case study used to evaluate the architecture in this paper are separated by a considerable distance. As a result, the routers do not interfere with each other, and can estimate the station where there is a guard with a rate of $100 \%$.

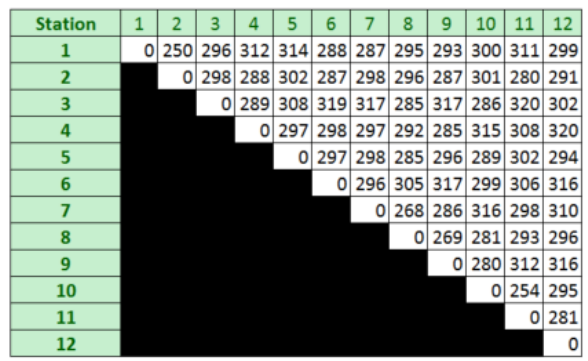

Fig. 4. Distances between stations in meters

To make the location process work correctly, it is necessary to link the metro stations with the Wi-Fi beacons that have been deployed in the case study. This relationship is implemented using a relational database, and the MAC addresses of the associated access points are stored for each station, as shown in Figure 6.

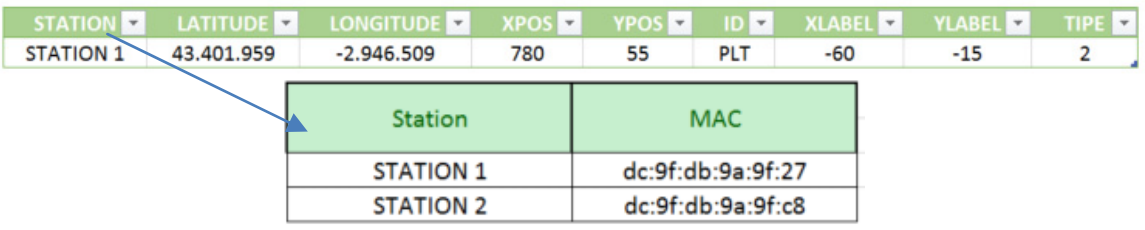

Fig. 5. Relationship between stations and routers

The central server stores the location data of the security guards in a database. These data are used to generate analysis and extract knowledge.

\section{$4 \quad$ Results and Conclusions}

The architecture presented in Section 3 has been installed in a subway network in the city of Bilbao in the north of Spain. The system infrastructure included 42 access points in 21 stations, and the security guards were equipped with 12 mobile devices, model GT-i9502 MTK6589, with an Android 4.2 operating system. The system was tested during one month and no anomalies were detected during the evaluation.

The central server was used by a supervisor to control the location of the security guards. To facilitate this task we developed a visualization tool that presents the location of the security guards in a very intuitive way. The central server implements monitoring services that are used by the supervisor to define alarms that are triggered when a security guard deviates from his or her usual route. 
Figure 7 shows a screenshot of the central server application which is installed in the central server and allows monitoring the security guards.

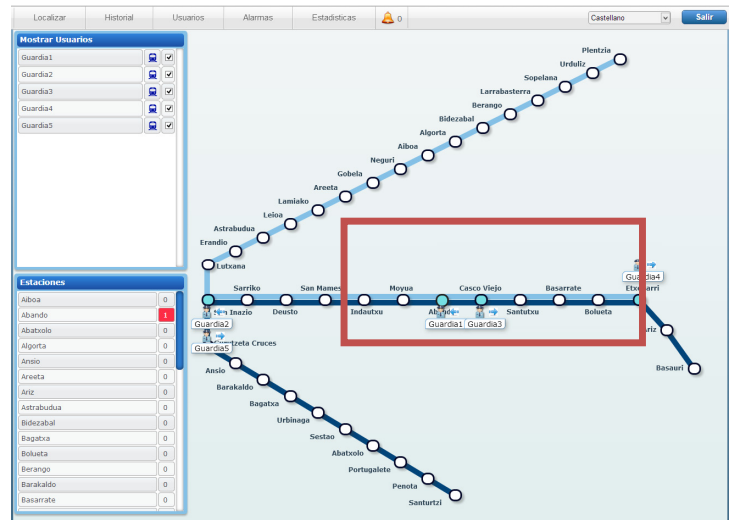

Fig. 6. Utility to view the positions

The users' location is updated in the visualization tool using Node.js technologie [9], an asynchronous platform that allows the development of scalable networks in a very quick way. The location of each security guard is asynchronously and immediately updated when a frame from a mobile device is received, without any refresh or request message required.

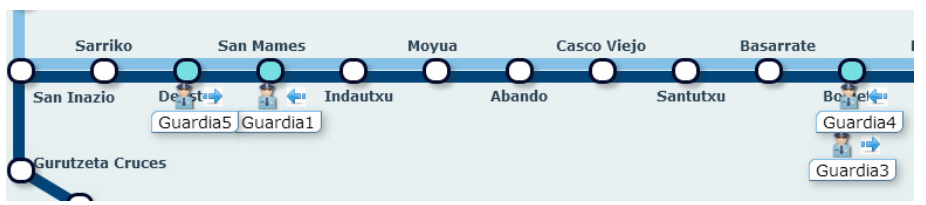

Fig. 7. Detailed View of lifeguard movements

System users have highlighted the ease of use and accuracy of the architecture. One of the most appreciated aspects among supervisors was the ability to generate multiple reports from the location data of the security guards stored in the database. For the supervisor it was of special interest to know the time spent by a security guard in a particular station, as shown in Figure 8.

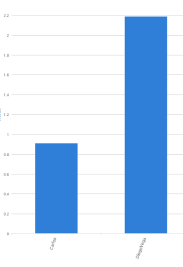

Fig. 8. Report Example 
Different tests were conducted to evaluate the battery consumption by comparing the proposed system with commercial systems. The use of a protocol with low latency and the avoidance of using conventional web services, yielded an average of $27 \%$ in terms of optimizing the battery life of mobile devices.

The results presented in this paper show that it is possible to implement a functional indoor locating system in an environment as complicated as subway stations, using wireless networks and with a reduced cost of deployment. It has been shown that the use of a M2M protocol provides quantitative battery savings in mobile terminals.

Finally, we believe that it is of interest to evaluate the system in a more complex environment and check whether there is any functional limitation on the number of users that can be supported by the application. This is our next challenge.

Acknowledgments. This work has been supported by the Ministry of Economy and Competitiveness "iHAS(Intelligent Social Computing for Human-Agent Societies)" financed by FEDER funds.

\section{References}

1. Anand, P., Siva Prasad, B.V., Venkateswarlu, C.: Modeling and optimization of a pharmaceutical formulation system using radial basis function network. International Journal of Neural Systems 19(2), 127-136 (2009)

2. Carnicer, J.M.C., García-Esnaola, M.: Lagrange interpolation on conics and cubics. Comput. Aided Geom. Design. 19, 313-326 (2002)

3. Chen, Y.-C., Chiang, J.-R., Chu, H.-H., Huang, P., Tsuid, A.W.: Sensor-Assisted Wi-Fi Indoor Location System for Adapting to Environmental Dynamics (2011)

4. Huang, C.N., Chan, C.T.: ligBee-based indoor location system by k-nearest neighbor algorithm with weighted RSSI. Procedia Computer Science 5, 58-65 (2011)

5. Tesoriero, R., Tebar, R., Gallud, J.A., Lozano, M.D., Penichet, V.M.R.: Improving location awareness in indoor spaces using RFID technology. Expert Systems with Applications 37(1), 894-898 (2010)

6. Hui, L., Darabi, H., Banerjee, P., Liu, J.: Survey of Wireless Indoor Positioning Techniques and Systems. IEEE Transactions on Systems, Man, and Cybernetics, Part C: Applications and Reviews 37(6), 1067-1080 (2007)

7. Web, http://stephendnicholas.com/archives/1217 (last visited January 14, 2014)

8. Tapia, D., Bajo, J., De Paz, J.F., Alonso, R.S.: Using Multi-Layer Perceptrons to Enhance the Performance of Indoor RTLS (2011)

9. Web, http: //nodej.s.org (last visited January 14, 2014)

10. Corchado, J.M., Fyfe, C.: Unsupervised neural method for temperature forecasting. Artificial Intelligence in Engineering 13(4), 351-357 (1999)

11. Fdez-Riverola, F., Corchado, J.M.: CBR based system for forecasting red tides. Knowledge-Based Systems 16(5), 321-328 (2003)

12. Tapia, D.I., Abraham, A., Corchado, J.M., Alonso, R.S.: Agents and ambient intelligence: case studies. Journal of Ambient Intelligence and Humanized Computing 1(2), 85-93 (2010)

13. Corchado, J.M., Lees, B.: Adaptation of cases for case based forecasting with neural network support. In: Soft Computing in Case Based Reasoning, pp. 293-319 (2001)

14. Corchado Rodríguez, J.M.: Redes Neuronales Artificiales: un enfoque práctico. Servicio de Publicacións da Universidade de Vigo, Vigo (2000) 
15. Bajo, J., Corchado, J.M.: Evaluation and monitoring of the air-sea interaction using a CBR-Agents approach. In: Muñoz-Ávila, H., Ricci, F. (eds.) ICCBR 2005. LNCS (LNAI), vol. 3620, pp. 50-62. Springer, Heidelberg (2005)

16. Fraile, J.A., Bajo, J., Corchado, J.M., Abraham, A.: Applying wearable solutions in dependent environments. IEEE Transactions on Information Technology in Biomedicine 14(6), 1459-1467 (2011)

17. Corchado, J.M., De Paz, J.F., Rodríguez, S., Bajo, J.: Model of experts for decision support in the diagnosis of leukemia patients. Artificial Intelligence in Medicine 46(3), 179200 (2009)

18. De Paz, J.F., Rodríguez, S., Bajo, J., Corchado, J.M.: Case-based reasoning as a decision support system for cancer diagnosis: A case study. International Journal of Hybrid Intelligent Systems 6(2), 97-110 (2009)

19. Tapia, D.I., Rodríguez, S., Bajo, J., Corchado, J.M.: FUSION@, a SOA-based multi-agent architecture. In: International Symposium on Distributed Computing and Artificial Intelligence 2008 (DCAI 2008), pp. 99-107 (2008)

20. Corchado, J.M., Aiken, J.: Hybrid artificial intelligence methods in oceanographic forecast models. IEEE Transactions on Systems, Man, and Cybernetics, Part C: Applications and Reviews 32(4), 307-313 (2002)

21. Corchado, J.M., Aiken, J., Rees, N.: Artificial intelligence models for oceanographic forecasting. Plymouth Marine Laboratory (2001)

22. Rodríguez, S., Pérez-Lancho, B., De Paz, J.F., Bajo, J., Corchado, J.M.: Ovamah: Multiagent-based adaptive virtual organizations. In: 12th International Conference on Information Fusion, FUSION 2009, pp. 990-997 (2009)

23. Tapia, D.I., De Paz, J.F., Rodríguez, S., Bajo, J., Corchado, J.M.: Multi-agent system for security control on industrial environments. International Transactions on System Science and Applications Journal 4(3), 222-226 (2008)

24. Borrajo, M.L., Baruque, B., Corchado, E., Bajo, J., Corchado, J.M.: Hybrid neural intelligent system to predict business failure in small-to-medium-size enterprises. International Journal of Neural Systems 21(04), 277-296 (2011)

25. De Paz, J.F., Rodríguez, S., Bajo, J., Corchado, J.M.: Mathematical model for dynamic case-based planning. International Journal of Computer Mathematics 86(10-11), 17191730 (2009)

26. Bajo, J., De Paz, J.F., Rodríguez, S., González, A.: Multi-agent system to monitor oceanic environments. Integrated Computer-Aided Engineering 17(2), 131-144 (2010)

27. Tapia, D.I., Alonso, R.S., De Paz, J.F., Corchado, J.M.: Introducing a distributed architecture for heterogeneous wireless sensor networks. In: Omatu, S., Rocha, M.P., Bravo, J., Fernández, F., Corchado, E., Bustillo, A., Corchado, J.M. (eds.) IWANN 2009, Part II. LNCS, vol. 5518, pp. 116-123. Springer, Heidelberg (2009)

28. Rodríguez, S., de Paz, Y., Bajo, J., Corchado, J.M.: Social-based planning model for multiagent systems. Expert Systems with Applications 38(10), 13005-13023 (2011)

29. Pinzón, C.I., Bajo, J., De Paz, J.F., Corchado, J.M.: S-MAS: An adaptive hierarchical distributed multi-agent architecture for blocking malicious SOAP messages within Web Services environments. Expert Systems with Applications 38(5), 5486-5499

30. Corchado, J.M., Bajo, J., De Paz, J.F., Rodríguez, S.: An execution time neural-CBR guidance assistant. Neurocomputing 72(13), 2743-2753 (2009)

31. Gómez, J., Patricio, M.A., García, J., Molina, J.M.: Communication in distributed tracking systems: an ontology-based approach to improve cooperation. Expert Systems 28(4), 288305 (2011)

32. Pavon, J., Sansores, C., Gomez-Sanz, J.J.: Modelling and simulation of social systems with INGENIAS. International Journal of Agent-Oriented Software Engineering 2(2), 196-221 (2008) 